\title{
INTEROPERABILITY TESTING SYSTEM OF TCP/IP BASED COMMUNICATION SYSTEMS IN OPERATIONAL ENVIRONMENT
}

Toshihiko Kato, Tomohiko Ogishi, Hiroyuki Shinbo, Yutaka Miyake, Akira Idoue and Kenji Suzuki

$K D D R \& D$ Laboratories, Inc.

Abstract Recently, TCP/IP protocols are widely used. Here, although the protocols are realized in operating systems and users of communication systems pay no attention to their detail, some system errors are actually reported. Since those errors occur only in specific communication situations, the interoperability testing by watching communication by a testing system is appropriate to detect them. This interoperability testing has the following requirements. Firstly, since the system that includes system errors is not unidentified, the testing system needs to check all communication systems attached to a network. Secondly, for the similar reason, all protocols in the TCP/IP protocol stack need to be checked. Thirdly, the testing system needs to discriminate operational failures, such as server down and mis-configuration of parameters in clients, from system errors, when it detects any problems in the communication. Based on these considerations, we have designed an interoperability testing system of TCP/IP based communication systems applied in an operational environment. This paper describes the detailed design of our testing system and the testing algorithm for DHCP (Dynamic Host Configuration Protocol), for which some system errors are reported.

Keywords: interoperability testing, TCP/IP, operational environment, multiple protocols

\section{INTRODUCTION}

Recently, the Internet becomes widely spread and many computer systems communicate with each other using TCP/IP protocols [5]. Here, the communication software is considered as a fundamental function such as an

The original version of this chapter was revised: The copyright line was incorrect. This has been corrected. The Erratum to this chapter is available at DOI: 10.1007/978-0-387-35516-0_20 
operating system, and most of users do not pay any attentions to its detail. Actually, commercial TCP/IP software is stable and, in most cases, users can access to WWW servers and send e-mails without any problems.

However, it is pointed out so far that there are some system errors of TCP/IP based communication systems. Examples are that some version of Solaris TCP software has problems in the initial value of retransmission timeout [4] and that some version of DHCP (Dynamic Host Configuration Protocol) [2] software of Macintosh has some bugs in the client operation [1]. Those errors are difficult to detect, because they occur only in exceptional situations such that the transmission delay increased dynamically due to network congestion, and that the combination of versions of DHCP software is one of special cases in a DHCP client and a DHCP server.

So far, the framework of protocol testing [3] identifies two testing approaches: the conformance testing and the interoperability testing. In order to detect the system errors in the TCP/IP communication systems described above, the interoperability testing approach is appropriate because they can be detected only while those systems are actually communicating with various systems and because the testing needs to be performed for relatively a long time.

In order to realize the interoperability testing in the operational environment, the following requirements need to be considered:

- Since any TCP/IP based communication systems attached to the network have the possibility to include system errors, all of which need to be the targets of testing.

- In the TCP/IP based communication systems, multiple protocols cooperate together to realize communication. They include the protocols related to specific applications, such as FTP and HTTP, and the protocols that play a supplementary role, such as DHCP and DNS (Domain Name System). There are possibilities that any of them have system errors, and therefore all of them need to be investigated.

- Users of TCP/IP based communication systems often suffer from failures due to the operational conditions, such as the server downs and the mis-configuration of parameters in clients. That is, the reason of a detected problem may be sometimes system errors and otherwise operational failures. Therefore, a testing system for an interoperability testing needs to check both possibilities described above.

Based on these considerations, we have designed an interoperability testing system, which detects system errors in the operational environment. Our testing system has the following features: 
- We adopt an approach of network monitoring [4]. That is, the testing system attached to a network captures all packets exchanged among communication systems in a network, and it looks for any problems.

- Our testing system detects both system errors and operational failures, and identifies their reasons.

- Our testing system analyzes the protocol procedures of communicating systems to detect system errors. It also estimates the network configuration, operational status of terminals and servers, and parameter settings, in order to detect operational failures [5].

We have designed an interoperability testing system for TCP/IP based communication systems. This paper describes the detailed design of our testing system. The next section and section 3 describe the design principles and the overall design of the testing system, respectively. Section 4 describes the testing algorithm for DHCP and the result of preliminary testing. We make a conclusion in section 5 .

\section{DESIGN PRINCIPLES}

(1) As shown in Figure 1, our testing system is attached to a LAN segment (subnet) and captures packets transmitted by communication systems such as terminals, servers and routers. If a network is divided into multiple subnets, one testing system is attached to an individual subnet.

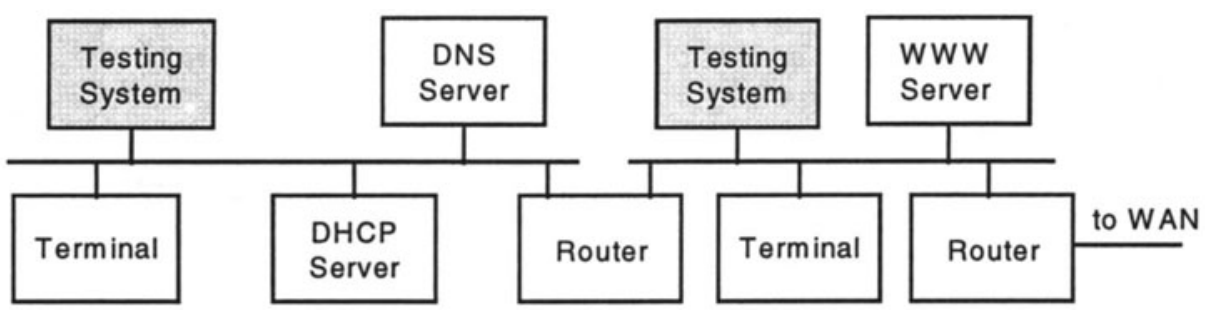

Figure 1. Network Configuration

(2) By using captured packets, our testing system performs the following:

- It identifies systems attached to the subnet automatically.

- It estimates the network configurations, such as the IP addresses of DHCP/DNS servers and routers (configuration estimation).

- For an individual captured packet, it emulates the protocol behavior of systems which sent or received the packet, and checks whether the behavior is conforming to the protocols (protocol emulation). If a 
problem is detected, it estimates that the problem comes from system errors or operational failures.

(3) As described above, the testing is required to operate for a long time. Therefore, our testing system needs to continue capturing packets transmitted by any system on the subnet and analyzing them to find system errors and operational failures. However, in the protocol emulation the correspondence between requests and responses needs to be examined and this requires our testing system to wait for responses when it detects requests. Therefore, we adopt the following approach:

- Our testing system keeps capturing packets in an online manner and saves them in a buffer, called the packet buffer.

- After a specific time of period (e.g. 120 seconds) has passed from the capturing of a packet, it considers that the related packets are also in the buffer and performs the protocol emulation for the packet.

(4) The first version of our testing system supports the interoperability testing the TCP/IP protocols including ARP, IP, ICMP, TCP, UDP, DHCP, DNS and SMTP. The protocol emulation is performed from lower layer to higher layer as shown in Figure2.

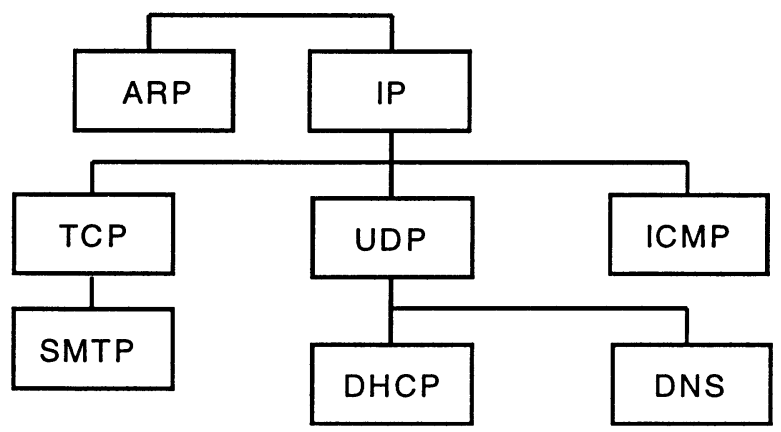

Figure 2. TCP/IP Protocols Handled in Testing System

\section{OVERALL DESIGN}

\subsection{Software Structure}

Figure 3 shows the software structure of our testing system. 

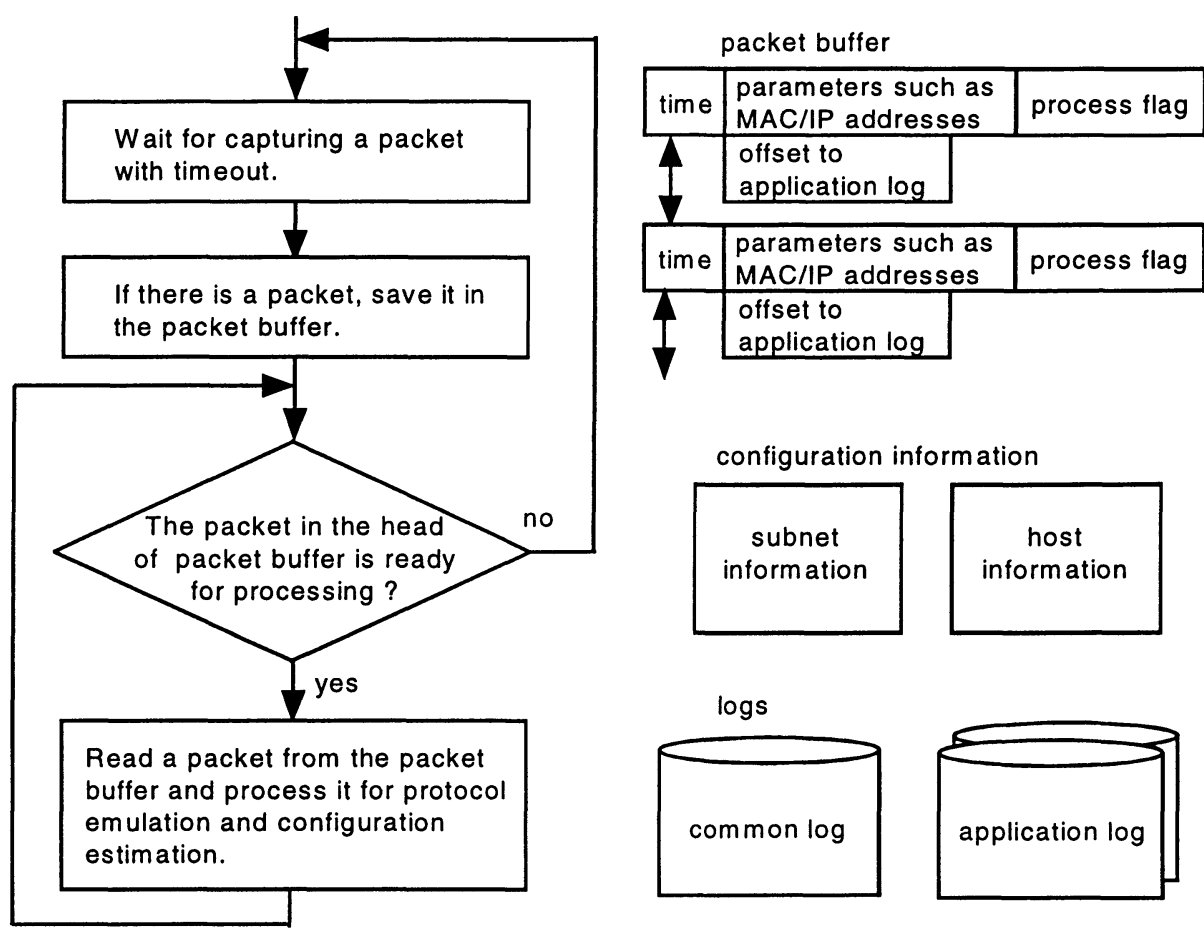

configuration information
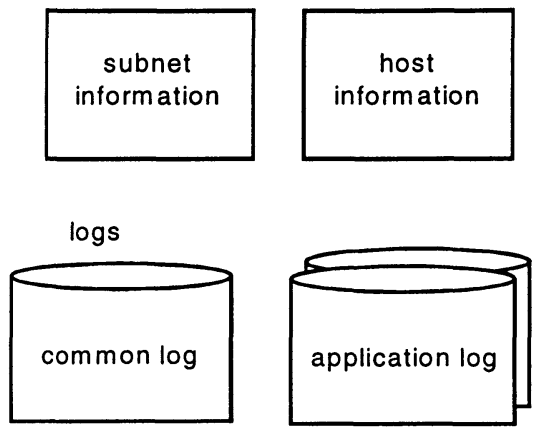

Figure 3. Software Structure

When the software captures a packet, it saves the information on it in the packet buffer. The packet buffer includes the time when this packet is captured and the parameters included in the packet. After that, the software checks if the packet saved in the head of the packet buffer is ready for processing, that is, a specific time elapsed after it was captured. If so, the software reads out the packet information from the buffer and performs the configuration estimation and the protocol emulation.

The result of configuration estimation is saved in the configuration information. This information consists of subnet information and host information. The subnet information includes the list of terminals and servers (hosts) attached to this subnet and the list of servers (DHCP, DNS and SMTP servers) which the hosts in this subnet have access to. The host information includes the information of an individual terminal or server, such as the MAC address and IP address.

The protocol emulation checks the behavior of the host sending the packet and detects operational failures and system errors. When some problems are detected, the results are displayed on the screen and stored in the logs. 
Basically, individual packets are handled one by one in the protocol emulation. However, for client-server type application protocols such as DHCP and DNS, the software pre-reads the corresponding response when a request is detected, and checks the mapping between the request and response. In order to realize this pre-reading, the log is prepared for individual application protocols and the packet buffer includes process flag indicating that the specific application protocol is processed before hand related to this packet.

\subsection{Information for Configuration Estimation}

As described above, the configuration information includes subnet information and host information. The subnet information includes the following information:

- Host list: each element includes its IP address and the pointer to host information.

- Router list: each element includes its IP address and working flag.

- DNS server list: each element includes its IP address, working flag and domain name table.

- DHCP server list: each element includes its IP address, working flag and available IP addresses.

- SMTP server list: each element includes its IP address, working flag and user list.

The host information includes following information.

- MAC address

- IP address

- State of IP address, which is used for inspecting duplicated IP address

- Working flag

- ARP cache table: each element includes IP address, hardware address and updated time

- List of routers the host uses: each element includes the router IP address, destination IP address and default router flag

- Use/nonuse of DHCP

- DHCP server address

When our testing system processes a captured packet, the information described above is updated. The configuration information helps our testing system to detect operational failures and system errors. 


\subsection{Overview of Protocol Emulation and Configuration Estimation Procedures}

This subsection gives overviews of protocol emulation and configuration estimation for ARP, IP and TCP as examples.

\subsubsection{Procedures for ARP}

(1) At first, our testing system deals with exceptional cases such as operational failures. For example, sender IP address is out of the subnet, which comes to be a configuration error in subnet mask, or a response to gratuitous ARP is detected, which comes to be a duplicated use of IP address.

(2) Our testing system identifies a host attached to the subnet from a pair of sender hardware address and sender IP address of ARP packets. If the identified host is not registered in the host list, it is registered. The testing system also detects an operational failure by duplicated use of IP address.

(3) Our testing system checks the behavior of ARP for corresponding ARP Request and ARP Reply and estimates its current ARP cache table of the host. If there are no responses to ARP Request, then the testing system recognizes that the target host is down, when it is registered in the subnet information. Otherwise, the testing system considers that the IP address of the host may be mis-configured at the sending host.

\subsubsection{Procedures for IP}

(1) Our testing system identifies a host attached in the subnet together with its hardware address and IP address from the source pair or the destination pair.

(2) When IP address represents a host out of the subnet, our testing system considers that the hardware address represents that of a next-hop router. If the router is not registered in the subnet information, it is registered. The testing system also updates the list of routers of the host.

(3) Then our testing system processes the user data of this packet according to the protocol ID. ICMP, UDP and TCP emulation is performed according to its value. If the protocol ID is not that of above, it produces an emulation result and performs the emulation of the next packet in the packet buffer. 


\subsubsection{Procedures for TCP}

(1) As for the processing of TCP, we depend on the Intelligent TCP analyzer, which we developed previously [4]. It emulates the TCP behaviors in the communication systems by using captured TCP packets. It maintains the state and internal variables such as "SND.NXT" and estimates TCP's internal procedures such as slow start and congestion avoidance algorithm. As a result, it detects the following system errors:

- No initial slow start

- No slow start after retransmission timeout

- Uninitialized congestion window

- Too small initial retransmission timeout

- Miscalculation of retransmission timeout

(2) If a captured TCP packet is "SYN" and "SYN+ACK" and the destination port of "SYN" is a well-known port of an application server, our testing system considers that the host focused on is working as a server. If the host is not registered in the list of the server, it is registered in the subnet information. Similarly, our testing system maintains the subnet information in response to the abrupt release of TCP and no response to "SYN".

(3) Then our testing system processes the user data of this packet according to the port number. Currently we are designing SMTP handling over TCP.

\section{DETAIL DESIGN FOR DHCP}

In this section, we describe more detailed design of our system by taking DHCP as an example.

\subsection{Overview of DHCP}

DHCP provides the function of allocating an IP address dynamically to a host. DHCP has the following procedures in which a host requests an IP address to a DHCP server:

(1) Allocating a new IP address:

A client that does not have its IP address broadcasts a DISCOVER message by setting all 0 address for its source IP address and all 1 address for its destination IP address. (Multiple) DHCP servers that can allocate an IP address respond to it with an OFFER message (see Figure 4).

The client which receives several OFFER messages chooses one server. It then broadcasts a REQUEST message by specifying the selected server ID and IP address provided by the server. In the end, the selected DHCP 
sever responds to it with an ACK message specifying the allocated IP address and its lease time. These messages are transmitted by UDP and the related messages are identified by the parameter, transaction ID.

It should be noted that OFFER and ACK messages will be broadcasted or unicasted according to the BROADCAST flag included in the DISCOVER and REQUEST messages respectively.

(2) Reusing a previously allocated IP address:

If a client remembers and wishes to reuse a previously allocated IP address, it sends a REQUEST message containing the IP address. If a DHCP server can allocate the requested IP address, it responds with an ACK message. There are three cases here. The first one is that a client requests the IP address during the lease time to the DHCP server which allocated it, which is called "RENEWING". In this case, the REQUEST message is unicasted. The second one is that a client broadcasts a REQUEST message to require the reallocation of the IP address during its lease time, which is called "REBINDING". The third one is that a client broadcasts a REQUEST message to expect the reallocation of the IP address after its lease time finished, which is called "INIT-REBOOT". These three cases are differentiated by the parameters included in the REQUEST message.

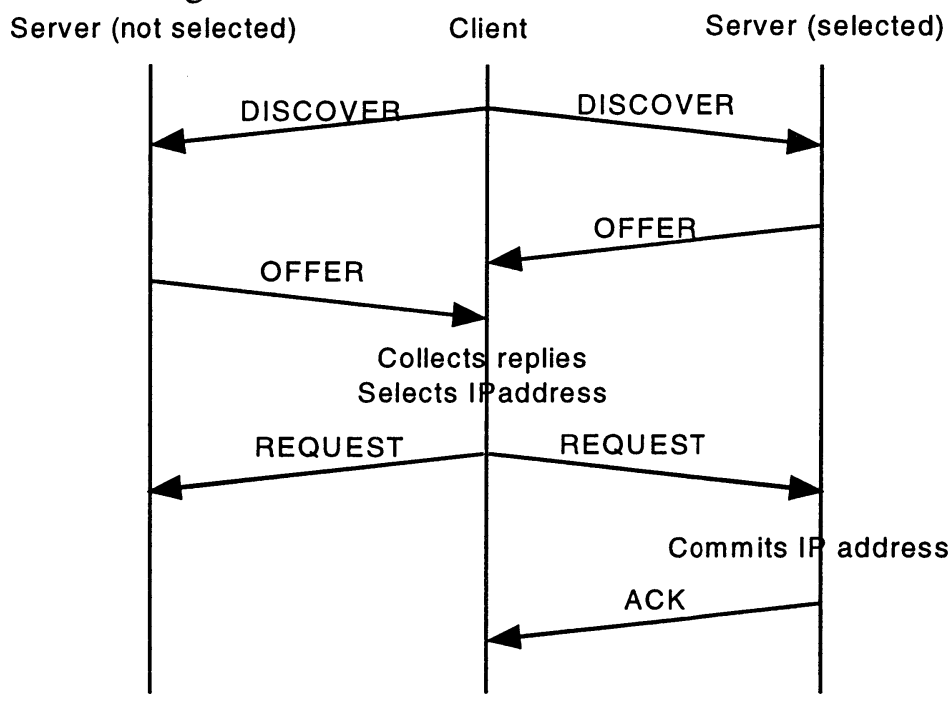

Figure 4. Sequence of DHCP When Allocating a New IP Address 


\subsection{Algorithm for DHCP Testing}

DHCP is a complex protocol due to the facts that it combines the unicast and broadcast communication and that the multiple procedures need to be realized by changing the combinations of parameters in the same message. Actually, some system errors related to DHCP are reported as described in section 1. In order to perform the interoperability testing of DHCP, we have designed the algorithm shown in Figures 5 and 6. This algorithm is for testing the case that a new IP address is allocated. It is summarized in the following way:

(1) When a packet being processed is a DISCOVER message, our testing system collects the related DHCP messages from the packet buffer. The related messages are those that have the same transaction ID as that of this DISCOVER message and whose process flag is not set. Those messages are copied in another buffer called the DHCP message buffer and their process flags in the packet buffer is set.

(2) The handling of DISCOVER message is to update the host and subnet information and check its format.

(3) Then the related OFFER messages are collected, and their format check and the update of the configuration information are performed. As described above, the BROADCAST flag in the DISCOVER message and the transmission of the related OFFER message that indicates broadcast or uni-cast need to be matched. So this matching is also examined. If there are no OFFER messages, a system error or an operational failure is reported. Here, it needs to be mentioned that the logs generated when our testing system handles pre-read packets are stored in the application log, and that the offset to the logs are stored in the packet buffer to be handled when the testing system processes the IP level later.

(4) Then, the related REQUEST messages are collected. If there are no REQUEST messages, a system error or an operational failure is reported. If there is one, the format check and the update of subnet information are performed. 


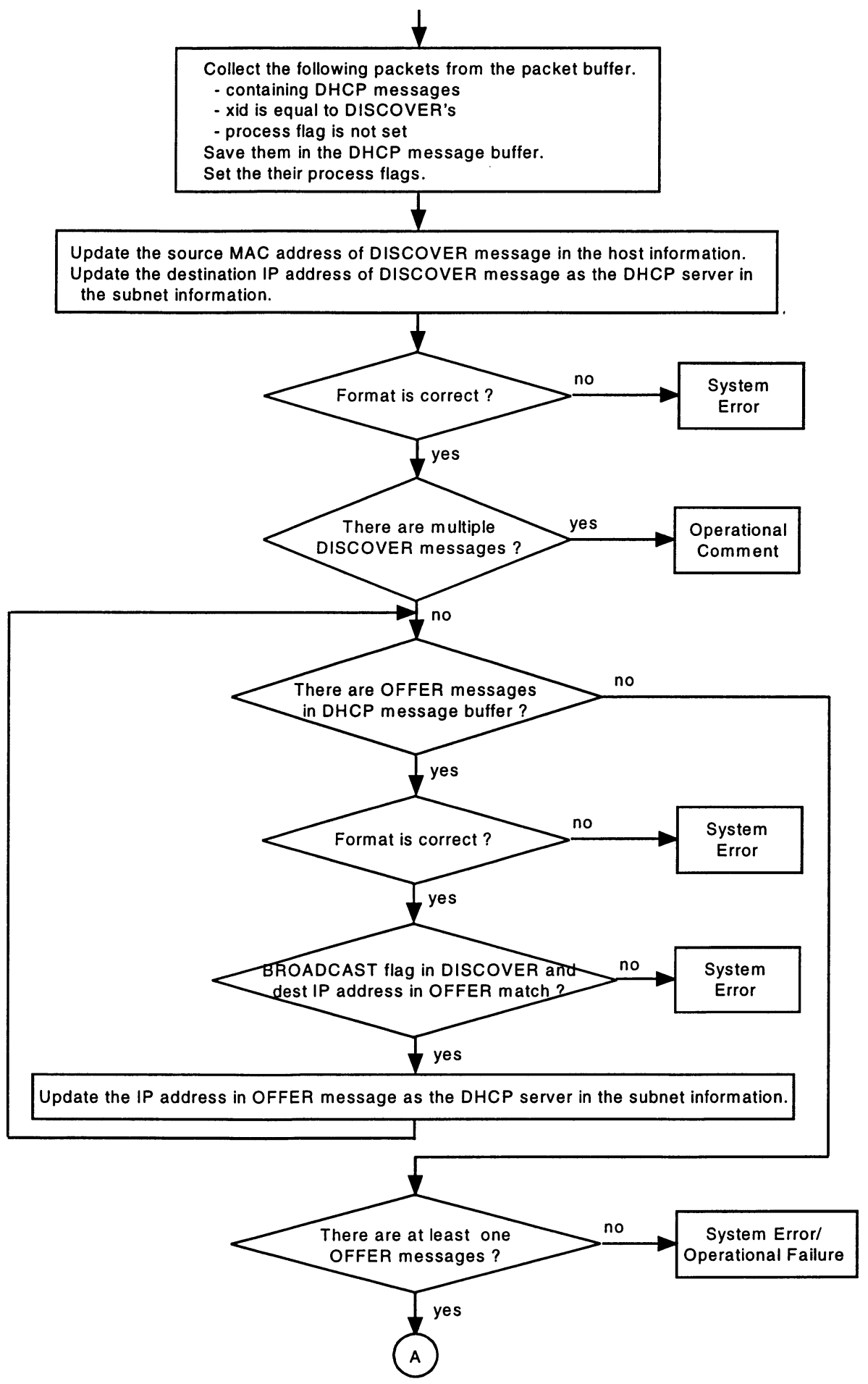

Figure 5. Algorithm for DHCP (1) 


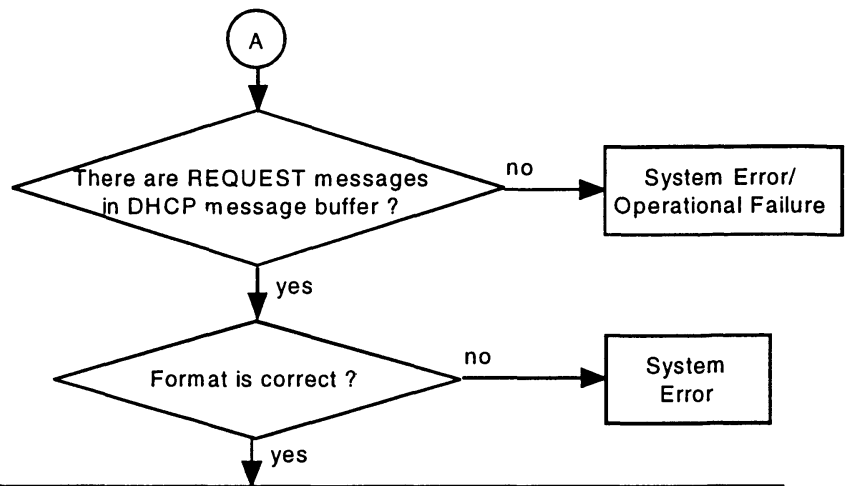

Update the IP address in REQEST message as the DHCP server in the subnet information.

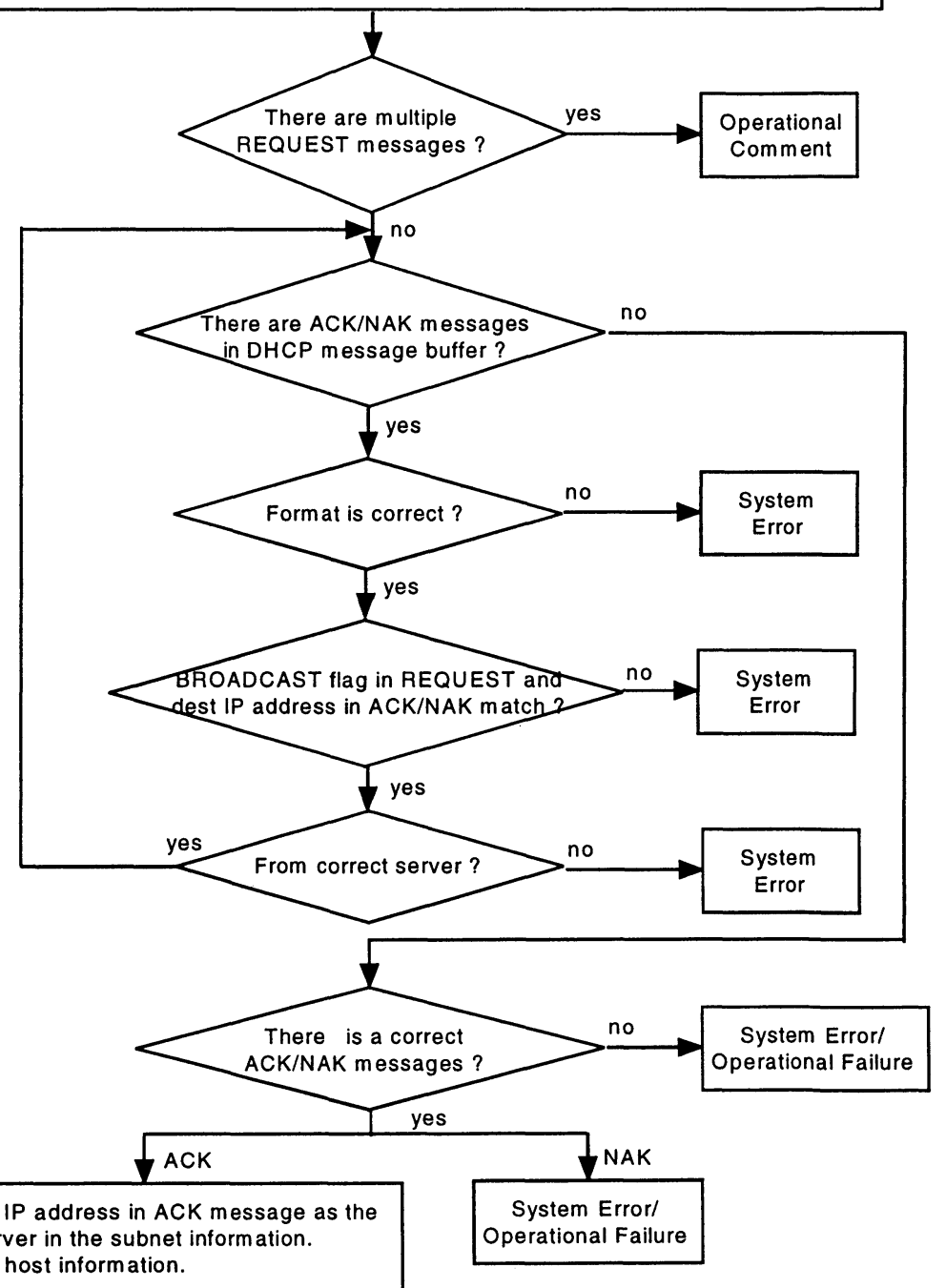

Figure 6. Algorithm for DHCP (2) 
(5) Then, the related ACK or NAK messages are collected and checked. The procedure is similar to that for OFFER messages. In addition to it, whether the ACK or NAK is sent by the DHCP server requested in the REQUEST message or not is checked. In the end, the host and subnet information is updated in the case of ACK message, and a system error or an operational failure is reported in the case of NAK message.

For the procedure for reusing a previously allocated IP address, the testing algorithm is designed similarly. In this case, the format check is important for discriminating RENEWING, REBINDING and INITREBOOT cases.

\subsection{Preliminary Experiment}

We have performed a preliminary experiment for the interoperability testing of DHCP based on the algorithm described in the previous subsection. As a result, we have found several system errors related to DHCP. The first example is shown in Figure 7. This is summarized in the as follows:

- When a Windows 98 personal computer is used as a DHCP client, it expects a broadcasted OFFER message even if it sends a DISCOVER message with its BROADCAST flag unset.

- When a Windows NT personal computer is used as a DHCP server, it broadcasts an OFFER message even if the BROADCAST flag in the corresponding DISCOVER message is unset.

- As a result, a Windows based client and server can communicate well.

- If a DHCP server works correctly such as the case of FreeBSD, that is, it unicasts an OFFER message, a Windows based client does not respond to it and retransmits a DISCOVER message. After the third OFFER message is received, it will respond to the OFFER message.

Figure 8 shows another example. This is summarized as follows.

- When a Macintosh personal computer is a DHCP client and an NTT router is a DHCP server, a Macintosh sends a DISCOVER message with a large value of IP address lease time. For that, an NTT router responds by an OFFER message with a smaller value of lease time.

- However, a Macintosh sends a REQUEST message with the same lease time as that of the DISCOVER message.

- As a result, the NTT router sends a NAK message.

- After that, a Macintosh again sends the same DISCOVER message and both of them continue the same procedure.

Our interoperability testing system can detect those system errors that will occur in an specific combination of communication systems. 

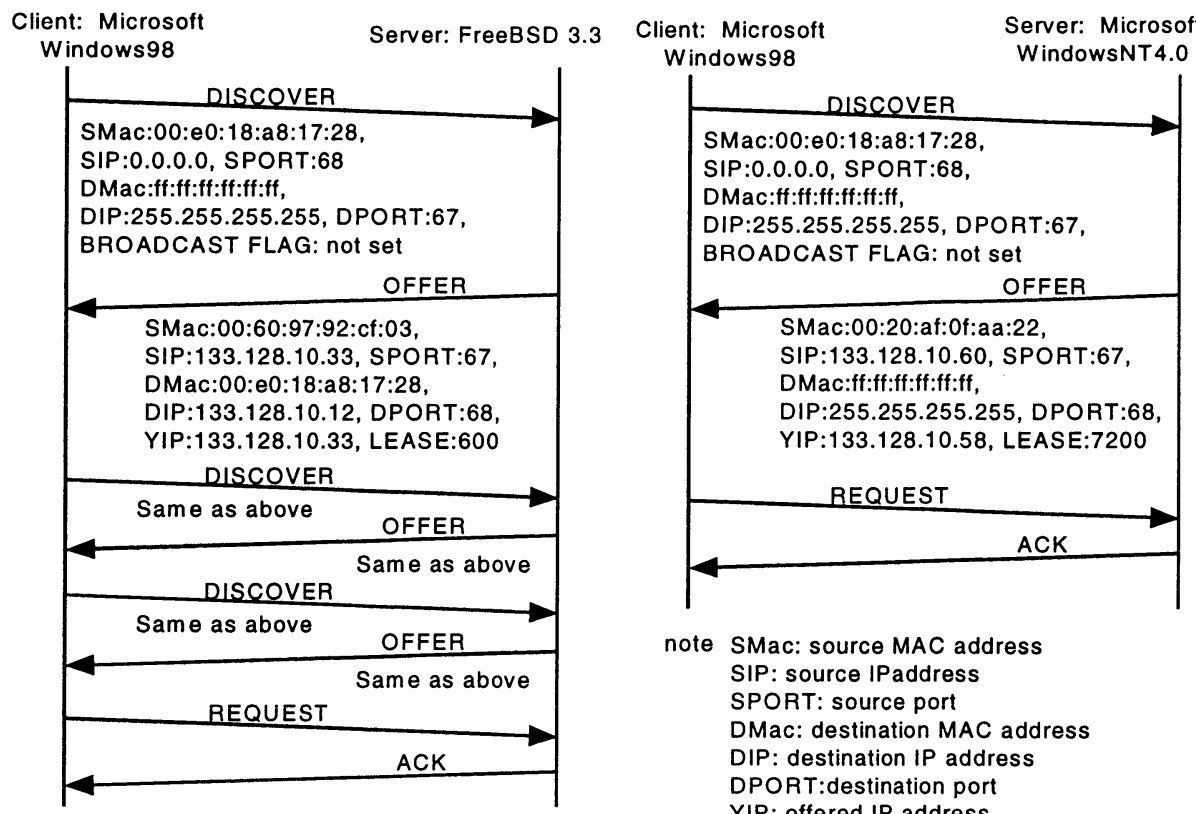

note SMac: source MAC address SIP: source IPaddress SPORT: source port DMac: destination MAC address DIP: destination IP address DPORT:destination port YIP: offered IP address LEASE: IP address lease time

Figure 7. Example of DHCP Error (1)

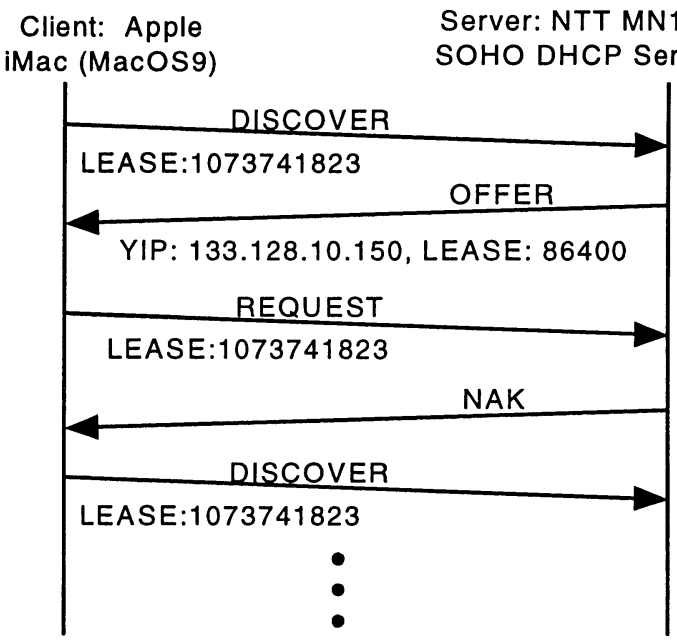

Figure 8. Example of DHCP Error (2) 


\section{CONCLUSIONS}

In this paper, we described the design of the interoperability testing system for TCP/IP based communication systems. Actually, most of TCP/IP based communication systems have only few system errors. However, some systems have bugs, which are difficult to find because they can be detected only in specific situations. In order to detect those errors, our testing system has the features to check any system attached to a network by considering the operational failures, such as server downs and parameter misconfigurations.

This paper gives a detailed design of our testing system, focusing on the DHCP testing by which it is possible that some systems have bugs. The results of the preliminary testing based on the design show that our testing system can detect system errors related to DHCP, which occur in a specific combination of clients and servers.

\section{References}

[1] Apple Care Tech Info Library, "Mac OS: DHCP Issues With Open Tansmport 2.5.1 and 2.5.2," http://til.info.apple.com/techinfo.nsf/ artnum/n25049, Nov. 1999.

[2] R. Droms, "Dynamic Host Configuration Protocol," RFC 2131, March 1997.

[3] ITU-T, "OSI Conformance Testing Methodology and Framework for Protocol Recommendations for CCITT Applications," X.290, 1988.

[4] T. Kato, T. Ogishi, A. Idoue and K. Suzuki, "Design of Protocol Monitor Emulating Behaviors of TCP/IP Protocols," Proc. of 10th IWTCS, Sept. 1997.

[5] T. Ogishi, A. Idoue, T. Kato and K. Suzuki, "A Protocol Analizer for Operational Testing of TCP/IP based Communication Systems," in Proc. of ICCC '99, Sept. 1999.

[6] V. Paxon, "Automated Packet Trace Analysis of TCP Implementations," in Proc. SIGCOMM '97, Aug. 1997.

[7] W. Stevens, "TCP/IP Illustrated, Vol. 1: The Protocols," Addison Wesley, 1994. 